\title{
ORIGINAL ARTICLE PhenoWorld: a new paradigm to screen rodent behavior
}

\author{
M Castelhano-Carlos ${ }^{1,2}$, PS Costa ${ }^{1,2}$, H Russig $^{3}$ and N Sousa ${ }^{1,2}$
}

Modeling depression in animals has inherent complexities that are augmented by intrinsic difficulties to measure the characteristic features of the disorder. Herein, we describe the PhenoWorld (PhW), a new setting in which groups of six rats lived in an ethological enriched environment, and have their feeding, locomotor activity, sleeping and social behavior automatically monitored. A battery of emotional and cognitive tests was used to characterize the behavioral phenotype of animals living in the PhW and in standard conditions (in groups of six and two rats), after exposure to an unpredictable chronic mild stress paradigm (uCMS) and antidepressants. Data reveal that animals living in the PhW displayed similar, but more striking, behavioral differences when exposed to uCMS, such as increased behavioral despair shown in the forced swimming test, resting/sleep behavior disturbances and reduced social interactions. Moreover, several PhW-cage behaviors, such as spontaneous will to go for food or exercise in running wheels, proved to be sensitive indicators of depressive-like behavior. In summary, this new ethological enriched paradigm adds significant discriminative power to screen depressive-like behavior, in particularly rodent's hedonic behavior.

Translational Psychiatry (2014) 4, e399; doi:10.1038/tp.2014.40; published online 10 June 2014

\section{INTRODUCTION}

Depression is a chronic recurring disorder that affects $\sim 20 \%$ of the population around the globe. ${ }^{1,2}$ It is a complex multidimensional disorder involving several signs and symptoms, such as loss of interest or pleasure in hobbies or activities, persistent sadness, helplessness, disturbed sleep and appetite, among others, varying with the patients. ${ }^{2}$ Modeling such a complex disorder in nonhuman species is remarkably difficult both from the conceptual perspective of how to reproduce mood disorders in such species, and also from an operational perspective of what to measure. The field has evolved not only to produce several animal models of the disorder, such as chronic stress paradigms, submitting animals to adverse early life events, olfactory bulbectomy and genetically modified animals or selective breeding, ${ }^{3}$ but also in the development of several behavioral tests that can measure different behavioral dimensions ${ }^{4,5}$ that are known to be relevant to depression. However, there are still limitations in our assessment of rodent's depressive-like behavior. ${ }^{6-10}$ One of the most obvious relates to the fact that hedonic behavior should be tested in an environment where the individual displays the motivation to do a particular action; so far, the tests we have to measure anhedonic behavior are based on the ratio of alternative choices and, in most cases, imply animal's isolation. Moreover, depressive-like behavioral tests (for example, forced swimming test (FST) and tail suspension test) are typically performed in different arenas than those where the animals live, which brings several confounding effects such as the reaction to a novel environment. Finally, in almost all testing conditions, the complexity of social interactions of living in large groups is lost as the animals are tested either individually or in small cohort interactive contexts.

In order to circumvent most of these limitations, we have conceptualized the PhenoWorld (PhW), a new paradigm to analyze rodents' behavior. In the PhW, groups of six rats with implanted radio-frequency identification (RFID) transponders lived in an ethological enriched setting, and had their feeding, locomotor activity, resting and social behavior automatically monitored. To validate the ability of this paradigm to screen depressive-like behavior, we have compared their depressive-like behavior in standard tests to that of animals living in standard conditions (in groups of six or pair-housed), after exposure to unpredictable chronic mild stress (uCMS), a validated animal model of depression, ${ }^{11,12}$ and following antidepressant therapy.

\section{MATERIALS AND METHODS}

\section{Animals and housing conditions}

Wistar Han IGS male rats, Specific Pathogen Free, with 7-8 weeks of age were purchased from Charles River Laboratories (Lyon, France), kept for 1 week in a quarantine room and then transferred to a conventional housing room. The animals were then subcutaneously injected with RFID transponders for individual identification (Yellow label transponder from Planet ID; ISO FDX-B Standard/manufacturer code 972) and randomly assigned to groups of six animals, housed in the following conditions: animals attributed to groups $\mathrm{PhW}$ or $\mathrm{Std}(6)$ were housed in a standard filter-topped transparent cage $610 \times 435 \times 215 \mathrm{~mm}\left(2065 \mathrm{~cm}^{2}\right.$ floor area) for six animals (ref. 2000P, Tecniplast, Buguggiate, Italy); and animals attributed to group $\mathrm{Std}(2)$ were pair-housed in a standard filter-topped transparent type III cage $425 \times 266 \times 185 \mathrm{~mm}\left(800 \mathrm{~cm}^{2}\right.$ floor area; ref. $1291 \mathrm{H}$, Tecniplast). The standard cage housing the six animals of PhW group was placed inside the central cage of the PhW, and the animals were allowed to adapt to it in a step-by-step approach, as further described under PhW setup below.

Two independent experiments were performed in control conditions (PhW, Std(6) and Std(2) groups), and two other independent experiments involved stressing the animals using a protocol of uCMS as described below (PhW+CMS, Std(6)+CMS and Std(2)+CMS groups).

All animals were maintained under standard laboratory conditions as follows: artificial $12 \mathrm{~h}$ light/dark cycle (lights on from 0800 to 2000 hours), with an ambient temperature of $21 \pm 1{ }^{\circ} \mathrm{C}$ and a relative humidity of 50-60\%; with corn cob bedding (Scobis Due, Mucedola SRL, Settimo Milanese, Italy) and sterile cardboard tubes as a housing refinement

\footnotetext{
${ }^{1}$ Neuroscience Research Domain, Life and Health Sciences Research Institute (ICVS), School of Health Sciences, University of Minho, Braga, Portugal; ${ }^{2}$ ICVS/3B's—PT Government Associate Laboratory, Braga/Guimarães, Portugal and ${ }^{3}$ TSE Systems GmbH, TSE Systems International Group, Bad Homburg vor der Höhe, Germany. Correspondence: Dr N Sousa, Neuroscience Research Domain, Life and Health Science Research Institute (ICVS), School of Health Sciences, University of Minho, Campus de Gualtar, Braga 4710-057, Portugal. E-mail: njcsousa@ecsaude.uminho.pt
}

Received 28 March 2014; accepted 22 April 2014 
(autoclaved paper rolls); the rats were given standard diet (4RF21, Mucedola SRL) and water ad libitum. Health monitoring was performed according to the Federation of European Laboratory Animal Science Associations (FELASA) guidelines, ${ }^{13}$ confirming the Specified Pathogen Free health status of sentinel animals maintained in the same room.

Animals were handled from beginning of the study by the same experimenter who performed all behavioral tests and also changed their cages, including adding new cardboard tubes once a week.

All experiments were performed according to the European Directive 2010/63/EU and the Portuguese regulations and laws on the protection of animals used for scientific purposes of the Ministry for Agriculture, Ocean, Environment and Spatial planning, which authorized the project where this study was included (authorization 9458 of 2011-05-06). The present study was also evaluated by the University of Minho ethics committee that approved it (process code SECVS 097/2013).

PhW setup. The PhW structure was developed for six rats in a developmental project established in collaboration with TSE Systems International Group (TSE Systems GmbH, Bad Homburg vor der Höhe, Germany). It provides housing and testing paradigms in an integrated structure by using automatic AnimalGates (AG) controlled by Intellimaze software (TSE Systems), combined with PhenoMaster software (TSE Systems) that allows control of accesses and automatic record of the animals' behavior in the PhW. The complete setup developed is presented
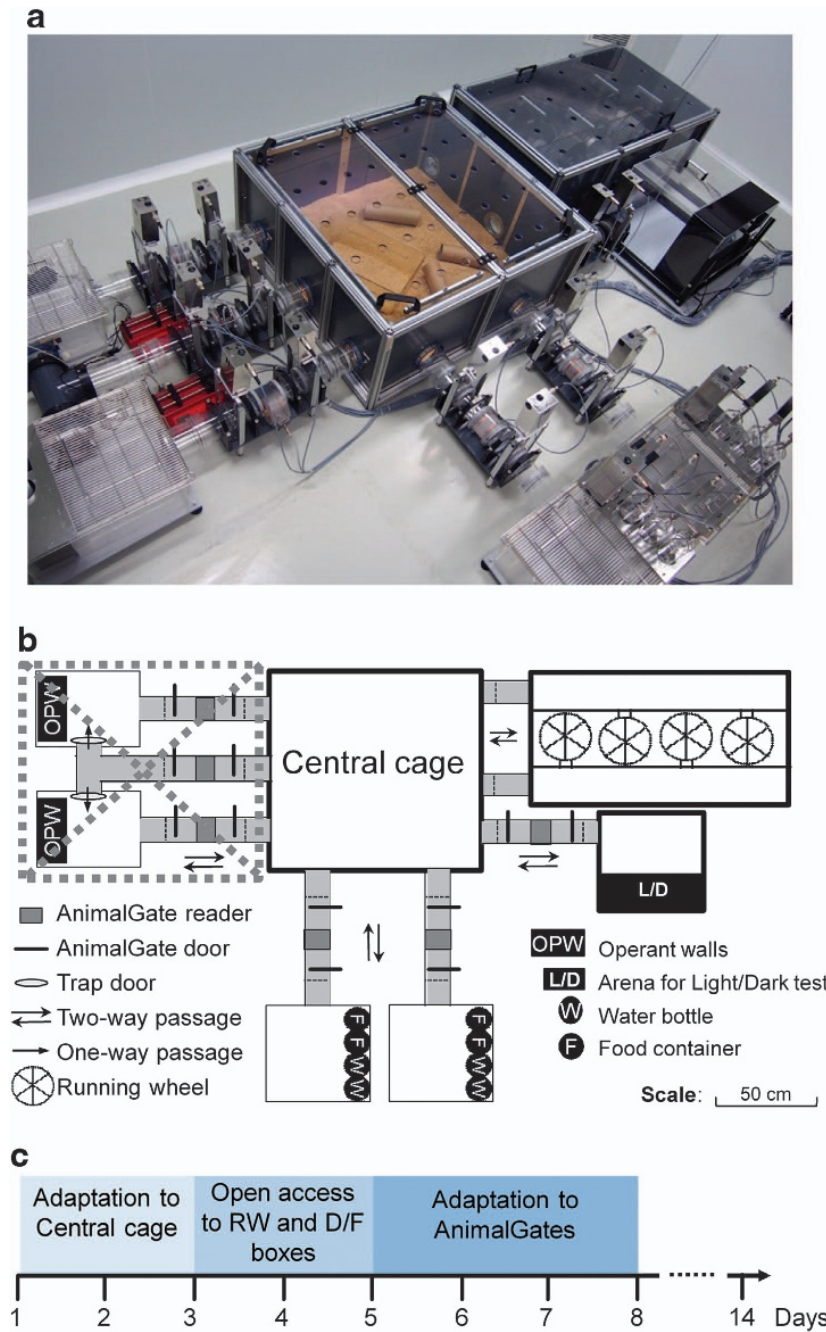

Figure 1. The PhenoWorld (PhW) paradigm: (a) photo of the PhW setup used; (b) scheme representing the PhW, with dashed gray square with a cross indicating the area that has not been used for the present study; and (c) adaptation period strategy used for the animals to adapt to the PhW. RW, running wheels; D/F, drinking/ feeding boxes. in Figure 1, with indication of the areas tested in this study. In brief, the area tested included a central cage of $1 \mathrm{~m}^{2}$, covered with corn cob bedding, and $50 \mathrm{~cm}$ height, connected to a box with four running wheels by means of two open access tubes and to two dinking/feeding boxes, each accessible by means of an AG. The central cage is also connected through one AG to an arena, for light/dark test, and three other AGs connect the central cage to two boxes equipped with operant walls, either directly or via a T-shaped access tube. This setup has the additional possibility to be linked to other boxes to test specific behaviors. All areas of the PhW have either perforated Plexiglas or stainless steel grids covering them, and all AGs or tube connections from the central cage can also be manually closed. As we aimed to characterize the behavior of animals living in the PhW and compare it with animals living in standard conditions, herein we used only the exercising and drinking/feeding areas of the PhW setup, and we analyzed the behaviors of the animals in standard testing conditions (Figure $1 \mathrm{~b}$ ). In order to register the data per animal, each AG and each running wheel is equipped with an RFID antenna/reader that recognizes the individual identification of the animals. As described for the standard cages, the PhW central cage contained cardboard tubes and a base of a standard type III cage, thereby giving the animals the possibility to climb or jump.

Cage change in the PhW consisted of closing the animals in the central cage for cleaning the surrounding boxes, and then while taking the animals out of the central cage for body weight measurements, dirty bedding was removed and the central cage was cleaned and new sterile bedding, a type III cage base and cardboard tubes were added to it.

Animals of PhW group were first placed inside the central cage of PhW in a standard cage for six animals (ref. 2000P, Tecniplast), as described previously. In the first 2 days, this cage was left open so the animals would adapt to the central cage area. On days 3 and 4, the standard cage with water and food was removed from the central cage of the PhW and the animals were given access to the running wheels and the drinking/feeding boxes, but with both doors of the AGs kept open for all animals. In the following days, the AGs for drinking/feeding boxes were opened and the animals had to learn to cross them, one at each time, to access water and diet (Figure 1c). Crossing the AG involved passing the first opened door, waiting few seconds in the center while the antenna reader detected the animal and the software closed the first door (behind the animal) and opened the second door (in front of the animal).

Animals' identification. The skin on the middle dorsal area of the animal, site of placement for the RFID transponder, was previously anesthetized by subcutaneous injection of $250-300 \mu \mathrm{l}(100 \mu \mathrm{l}$ per $100 \mathrm{~g}$ body weight) of $0.5 \%$ Lidocaine (from 2\% Lidocaine solution, B.Braun Medical Lda, Queluz de Baixo, Portugal), using a $25-\mathrm{G}$ ( $25 \mathrm{~mm}$ long) needle. A RFID transponder, $12 \mathrm{~mm}$ long $\times 2.12 \mathrm{~mm}$ diameter, $0.09 \mathrm{~g}$ weight, covered with Bio Glass 8625 and inserted in a $2.6 \mathrm{~mm} \times 32 \mathrm{~mm}$ needle, was then subcutaneously injected with the help of a transponder injector (injector and Yellow label transponder from Planet ID; ISO FDX-B Standard/manufacturer code 972).

\section{Biometric data}

Body weight. Body weight was measured every week along the course of the experiment using a dynamic animal weighing balance (Explorer Pro EP2102CM, Ohaus Europe, Nänikon, Switzerland).

Water and food intake. For comparison, water and food intake was calculated per cage by weighing the water bottles and the food pellets added to each cage before and after consumption, every other day, during a week period at baseline and at the end of the experimental period.

In the case of PhW group, water and food intake were also automatically registered by PhenoMaster software every 5 min during all the time the animals lived in the PhW. Intellimaze software (TSE Systems) controlled the AGs and registered the RFID transmitter of each animal going to a drink/ feed box at a given time; this information was processed by the PhenoMaster software (TSE Systems), that registered the water and food sensor values and automatically calculated the corresponding amount consumed though allowing to obtain drink/feed intake values per animal, and registering the exact periods of consumption for each animal.

Plasma corticosterone. In order to measure the plasma corticosterone levels, small blood samples were collected from the tail vein of the animals by using a sterile scalpel blade (one drop, corresponding to $\sim 50 \mu \mathrm{l}$ of blood per animal in each time point) at the following time points: immediately after the light of the housing room was switched on 
(designated as CORT (8 h)), and immediately after the light of the housing room was switched off (designated as CORT $(20 \mathrm{~h})$ ). The samples were collected in an adjacent, separate, dedicated room within 2 min of retrieving the animals from their home cage. Samples were stored at $4{ }^{\circ} \mathrm{C}$ until centrifugation for $10 \mathrm{~min}$ at 13000 r.p.m. (Biofuge Fresco, Heraeus, Osterode, Germany), and blood plasma was then separated and stored at $-80^{\circ}$ until analysis. Plasma corticosterone levels were determined using a commercial radioimmunoassay kit (ImmuChem, MP Biomedicals, LLC, Orangeburg, NY, USA), following the protocol of the supplier. Each sample was analyzed in duplicates.

\section{Unpredictable chronic mild stress}

We used a CMS model of depression in the present study. The stressed animals, corresponding to groups PhW $+\mathrm{CMS}$, Std(6)+CMS and Std(2)+CMS, were submitted to a slightly modified version of an uCMS protocol. ${ }^{12,14}$ In brief, it consisted of chronic exposure to unpredictable mild stressors, applied randomly every 7 days of the week over a 6-week period. The stressors applied were as follows: confinement to a restricted space for $1 \mathrm{~h}$; placement in a tilted cage $\left(30^{\circ}\right)$ for $3-4 \mathrm{~h}$; exposure to sudden noises for 3-4 h; housing on wet bedding for $8 \mathrm{~h}$; overnight illumination; housing in a type III cage with cold water instead of bedding for $1 \mathrm{~h}$; cage change with other group of animals; $12-14 \mathrm{~h}$ food deprivation followed by exposure to inaccessible food for $1 \mathrm{~h}$; water deprivation for $12-14 \mathrm{~h}$ followed by exposure to an empty bottle for $1 \mathrm{~h}$; and reversed light/dark cycle for 24 or $48 \mathrm{~h}$

\section{Behavioral evaluation}

The animals were handled along all experimental period by the same researcher who performed all behavioral test described below.

Home-cage behavior. In order to analyze the animals behavior and welfare in different living conditions as well as when using the depression model by uCMS, we used a scan sampling analysis of home-cage behavior. ${ }^{15}$ The animals were pen marked on their tail on the previous day. On the day of home-cage behavior recording, on the week after the period corresponding to UCMS protocol, the animals were videotaped undisturbed in their home cage. The videos were then analyzed and several patterns of behaviors were scored, as present or absent, for each animal at minutes $8,16,24$ and 32 of a 35-min home-cage observation period. The following two observation sessions were scored: one in the light phase (between 0830 and 1130 hours) and another one in the dark phase (between 2030 and 2330 hours) of the light/dark cycle.

All videos were scored by the same observer. A second observer scored part of the videos to control for a possible bias. The patterns of behavior analyzed included sleep, locomotion, self-maintenance (grooming and licking its own body), interaction with objects in the cage (for example, cardboard tubes), digging in the bedding and social interaction (including social investigation, allogrooming, play behavior and clump (two or more animals sleeping/resting in contact with each other)). ${ }^{15,16}$

In the case of animals living in the PhW, behaviors registered automatically by PhenoMaster software, per animal, were also analyzed. Those included consumption of water and food and different parameters of running wheels activity (time and distance run, number of runs, maximum length of a run and speed of each run).

For more details on the behavioral parameters analyzed by scan sampling of home-cage videos view Supplementary Tables S1 and S2.

Sucrose and saccharin preference tests. To evaluate hedonic behavior, sucrose preference tests (SPTs) were performed at baseline and over the 6 weeks of exposure to uCMS. To test sucrose preference, animals that were food and water deprived for 20-23 $\mathrm{h}$ were presented with two preweighed bottles containing $2 \%$ sucrose solution (Saccharose: cat. \# 141621.1211, Panreac Química SLU, Barcelona, Spain) or just autoclaved tap water for a period of $1 \mathrm{~h}$. Sucrose preference was calculated according to the formula: sucrose preference $=$ (sucrose intake/(sucrose intake+water intake) $\times 100$, as previously described. ${ }^{17}$ Anhedonia is defined as a reduction in sucrose preference relative to baseline levels.

Animals were tested for sucrose preference in their active period - tests started between 2000 and 2200 hours. For the test, each animal from Std (2) or Std(6) groups was placed alone in a standard type III cage, where the water bottle and the $2 \%$ sucrose bottle were positioned in the place usually used for the diet. The relative position of the bottles (left or right) was changed in each test performed. As there were only two drink/feed boxes in the PhW setup, for a group of six animals living there, SPT tests had to be made for two animals in three different time points: around 2000, 2100 and 2200 hours. The same three time points were used for each group of six animals even when the animals were housed in standard cages. The order of the animals performing the first, second and third SPT test in each test day was randomly changed along the study period.

In the case of PhW group, the animals were manually closed on the central cage 1.5- $2 \mathrm{~h}$ before the first test time to avoid them from exercising on the running wheels just before the test.

In order to confirm that we were evaluating hedonic behavior of the animals, we also performed the same preference test but used saccharin (SacchPT) to control for the caloric value of the drinking solution. As optimal $0.2-0.4 \%$ saccharin solutions have been shown to be 'isoprefered' by rats to $2-4 \%$ sucrose solutions, ${ }^{18}$ we used a $0.2 \%$ saccharin solution (Saccharin sodium salt hydrate >98\%; cat. \# S1002, Sigma-Aldrich Chemie $\mathrm{GmbH}$, Steinheim, Germany). Rats' preference for saccharin has been used as a hedonic measure and CMS has been shown to cause anhedonia which have been measured by the reduction in sucrose or saccharin intake. ${ }^{19}$

After the SPT or SacchPT the animals went back to their original cage and were given food and water ad libitum. Animals living in the PhW were allowed free access to the drink/feed boxes with inactivated AG for the rest of the dark period after SPT or SacchPT.

Forced swimming test. The FST was used to evaluate behavioral despair. In brief, $24 \mathrm{~h}$ after a pre-test session, rats were placed again in transparent glass cylinders $(62 \mathrm{~cm}$ height and $25.5 \mathrm{~cm}$ diameter) filled with water $\left(23-24^{\circ} \mathrm{C}\right.$, depth $50 \mathrm{~cm}$ ) for a period of $5 \mathrm{~min}$. The sessions were video recorded and behavioral responses were scored offline using Kinoscope, an open source computer program available on the website http:// sourceforge.net/projects/kinoscope/?source = directory, and developed by Dalla et al. ${ }^{20}$ During the test period, the experimenter left the test room.

Behavioral despair was defined as an increase in the time of immobility and a decrease in latency to immobility. An animal was considered immobile when floating without moving or while performing the minimum activity required to keep the head above the water ${ }^{21,22}$ (usually a slight movement of one paw).

Light/dark box test. The light/dark box test was used to evaluate anxiety behavior. In our study, we used an acrylic box consisting of one light area about $2 / 3$ of total arena (51 (length) $\times 34.5$ (width) $\times 39.5$ (height) $\mathrm{cm}$ ) illuminated by a white fluorescent lamp with an intensity of 400 lux at the arena floor level (11 W/830 OSRAM DULUX S; OSRAM, Berlin, Germany), and a smaller dark box about $1 / 3$ of total arena (51 (length) $\times 16.5$ (width) $\times 39.5$ (height) $\mathrm{cm}$ ) at 1-6 lux (the higher intensity was measured at the entrance from the illuminated area at floor level). The acrylic box $(51 \times 51 \times 39.5 \mathrm{~cm})$ is surrounded by an infrared sensors frame for automatic registration of the animals' movements during the period of test, including rearing (PhenoMaster ActiMot system, TSE Systems, Germany).

Animals living in standard cages were brought to the test room $1.5-2 \mathrm{~h}$ before the test, whereas animals living in the PhW stayed in their homecage setup to which the light/dark test arena was attached. In the case of PhW group, the access to the running wheels cage was manually closed 1.5-2 $\mathrm{h}$ before the first test time.

For the light/dark box test, rats were placed in center of the illuminated area, facing the entrance of the dark area, and were allowed to explore the box for $5 \mathrm{~min}$. The apparatus was carefully cleaned with $10 \%$ ethanol solution and was allowed to dry between tests of different animals.

For characterizing the anxiety of the animals in each housing and stress condition, we analyzed latency for the first entry in the dark box and time spent in the light and in the dark areas. Increased anxious state was characterized by a decrease in latency to enter the dark area and an increase in the time spent in it. ${ }^{23,24}$

Novel object recognition test. We tested recognition memory of the animals using an adapted version of the non-matching-to-sample learning task as previously described. ${ }^{25}$ To minimize the confounding influence of spatial or contextual factors and the presence of stimuli in the testing room, we used a black box $(50 \times 50 \times 150 \mathrm{~cm})$ that could be adapted inside the open-field arena included in the PhW setup (acrylic box of $51 \times 51 \times 39.5 \mathrm{~cm}$; TSE Systems). The box was illuminated by a white 
fluorescent lamp at an intensity of 100 lux (11 W/830 OSRAM DULUX S) measured at the floor level.

Animals living in standard cages were transported to the test room $2 \mathrm{~h}$ before the test. All animals were acclimatized to handling and transport in the test room every day and two days before starting the test. Day 0 of the novel object recognition test (NOR) consisted in adaptation to the NOR test box by placing the animal in the test apparatus without objects for about 10 min. On day 1, a first 10-min trial was performed with two similar objects (sample trial) placed near each corner of the box, opposite to the wall where the animals started the test. On day 2 of the NOR test, $24 \mathrm{~h}$ after the first sample trial, a 3-min choice trial was performed using the test configuration of the first trial but replacing one of the sample objects by an unfamiliar object (the novel object). The position to place the animals in the test box in the beginning of each trial was in the mid-point of the wall opposite to the wall with objects and turned back to the objects. The experimenter always left the room during each trial. The test box and objects were carefully cleaned with $10 \%$ ethanol solution and were allowed to dry between tests of different animals. Videos of day 2 trials were analyzed using Kinoscope..$^{20}$ Exploration of an object was defined as directing the nose to the object at a distance of $<2 \mathrm{~cm}$ or touching it with the nose/mouth and walking on it. Rearing near the object was not counted as exploring the object.

Considering exploration of novel objects an expected behavior in rats, we used the percentage of time spent with the novel object during the choice trial as an indication of memory for the familiar object. The total time spent with objects gave information on the general exploratory activity of the animals in the NOR test context.

Antidepressant treatment. Another set of controls and stressed animals were prepared to assess the antidepressant effects of fluoxetine (fluoxetine hydrochloride: ( \pm )-N-Methyl-3-(p-trifluoromethylphenoxy)-3-phenylpropylamine hydrochloride; cat. \# 59333-67-4, Kemprotec Limited, Cumbria, UK), a selective serotonin reuptake inhibitor. Fluoxetine was administered by intraperitoneal injection daily for the last 2 weeks of the above-described uCMS protocol, at a dose of $10 \mathrm{mg} \mathrm{kg}^{-1}$. At the end of the treatment, animals were behaviorally assessed.

\section{Statistical analysis}

Sample size was determined based on the following two assumptions: the PhW limitations in number of animals (six per experiment), and the calculated adequate number of animals considering a medium effect size $(f=0.25)$, a type I error $a=0.05$ and a statistical power (1- type II error) of 0.8 . In practice, we ended up using a total of 108 animals.

Data obtained during the course of the study, such as body weight, SPT and SacchPT curves, and different time points of plasma corticosterone levels were analyzed using repeated measures analysis of variance (ANOVA).

Quantitative data obtained for water and food consumption per day, for each plasma corticosterone measurement, during home-cage behavior scan sampling for the several parameters analyzed, regarding light/dark box test, FST and NOR test, and for water and food consumption, as well as running wheels activity per day in the PhW, were analyzed by one-way ANOVA at baseline considering housing as between-subjects factor and also by two-way ANOVA considering housing and stress as betweensubjects factors, 6 weeks after baseline. Whenever appropriate, post hoc multiple comparisons between experimental groups were performed using Bonferroni's test. When a significant interaction between housing and stress was identified, independent samples $t$-test were performed to identify significant differences between control versus stressed animals, living in the same housing conditions.

The effect of antidepressant treatment on stressed animals was evaluated by two-way ANOVA, considering housing and antidepressant as between-subject factors. When evaluating the effect of antidepressant in parameters obtained only for animals living in the PhW (automatic recordings from software), independent samples $t$-test were performed to verify significant differences between fluoxetine-treated versus untreated stressed animals.

Statistical test assumptions were validated for all the analysis. Normality was assessed using Kolmogorov-Smirnov test complemented with the evaluation of skewness (SK), kurtosis (K) as well as histograms. Outliers exclusions were based on the $Z$-score criteria $Z>|3|$.Results showed that when normality was not accomplished with Kolmogorov-Smirnov test, all variables presented absolute SK and $K$ below 1 , which means that the distribution was close to normal, so we assumed using parametric tests for statistical analysis. Sphericity assumption (for repeated measures) and homogeneity of group variances were also verified and statistical analyses were made accordingly.

When parametric test assumptions were not verified, we used nonparametric tests as an alternative for statistical analysis, such as Kruskal-Wallis test (instead of one-way ANOVA), non-parametric ANOVA (using ranks; instead of two-way ANOVA) and Friedman test (instead of repeated measures). As significances for all results were the same, we opted to present all results from the parametric analysis.

All data are presented as means \pm s.e.m. In all cases, statistical significance was set at $P \leqslant 0.05$ (two-sided). Statistical analysis was performed using IBM SPSS statistics 20.0 (IBM Corporation, Armonk, NY, USA).

\section{RESULTS}

Housing conditions adaptation

At 11-12 weeks of age, animals started the PhW adaptation (Figure 1c) before any behavioral test was performed (for details see Supplementary Information and Video). After 2 weeks of adaptation period, all groups of animals were using their home cages routinely and were also used to the handling procedures.

\section{Biometric data}

Body weight variation. Analysis of body weight and body weight variation during all the time course of the experiment showed a general effect of time $\left(F_{(11,858)}=481.162, P<0.001\right.$, with older animals weighing more) and an effect of stress (loss of body weight) $\left(F_{(1,78)}=68.339, P<0.001\right.$; Figure $\left.2 a\right)$. Body weight gain analysis revealed an effect of housing conditions and of stress $\left(F_{(2,78)}=7.926, P=0.001\right.$ and $F_{(1,78)}=9.776, P=0.002$, respectively), and Bonferroni's post hoc test indicated a significant difference between animals housed in the PhW and animals housed in standard cages of six $(\operatorname{Std}(6))$ or pair-housed animals (Std(2); $P=0.004$ and $P<0.001$, respectively). When analyzing separately, the animals before and after the uCMS period (between 11 and 16 weeks of age and between 16 and 22 weeks of age, respectively), statistically significant differences were found in the first period between PhW and Std(6) or Std(2) animals, with PhW animals gaining less weight during the adaptation period and recovering after that $\left(F_{(2,81)}=33.673, P<0.001\right.$; Bonferroni's post hoc, $P<0.001)$. After starting uCMS, stressed animals gained less weight than control animals $\left(F_{(1,78)}=27.794, P<0.001\right.$; Figure 2b).

Water and food intake. No differences were observed in the total water and food intakes per day per animal as measured by weighing water bottles and food pellets before and after consumption. After adapting to their housing conditions, all animals consumed similar amounts of water and food at baseline, with 14-15 weeks of age, and at the end of the experimenta period, with 22-23 weeks of age. Even though weighing less than controls, animals that have been submitted to UCMS protocol presented the same water and food intake values per day per animal at 22-23 weeks of age (Figures $2 c$ and d).

Plasma corticosterone. Baseline plasma corticosterone levels confirmed a significant difference between different time points (Figure 2e), being lower when lights went on (CORT $(8 \mathrm{~h})$ ) and higher when lights went off (CORT $(20 \mathrm{~h})) \quad\left(F_{(1,78)}=225.924\right.$, $P<0.001)$. The same was verified for plasma CORT levels after CMS (data not shown)

One-way analysis of basal plasma corticosterone revealed a significant effect of housing for both CORT $(8 \mathrm{~h})\left(\mathrm{F}_{(2,80)}=9.891\right.$, $P<0.001)$; Bonferroni's post hoc showed a significant difference between PhW animals that presented the lowest corticosterone levels at $8 \mathrm{~h}$ and both standard housed groups $(P<0.001$ and $P=0.026$, for Std(6) and Std(2), respectively). For CORT levels at 

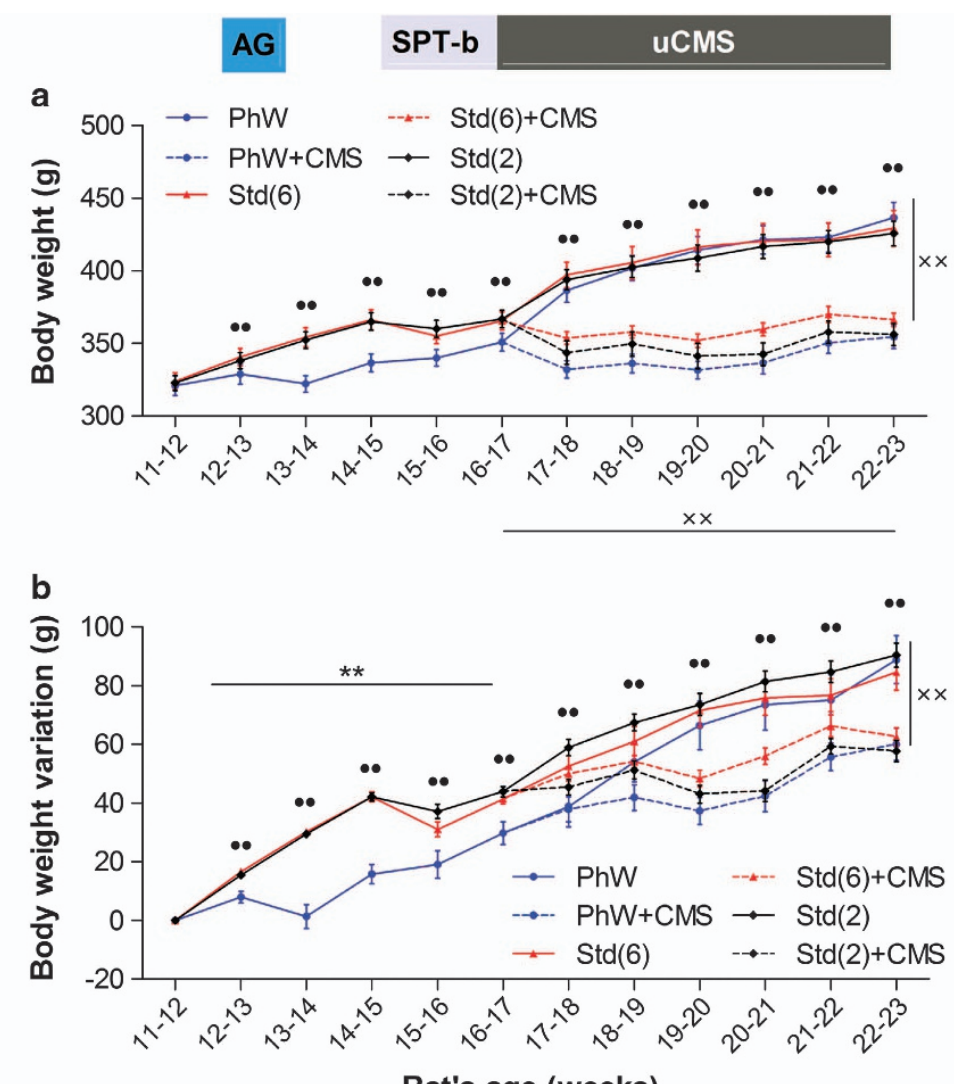

\section{Rat's age (weeks)}
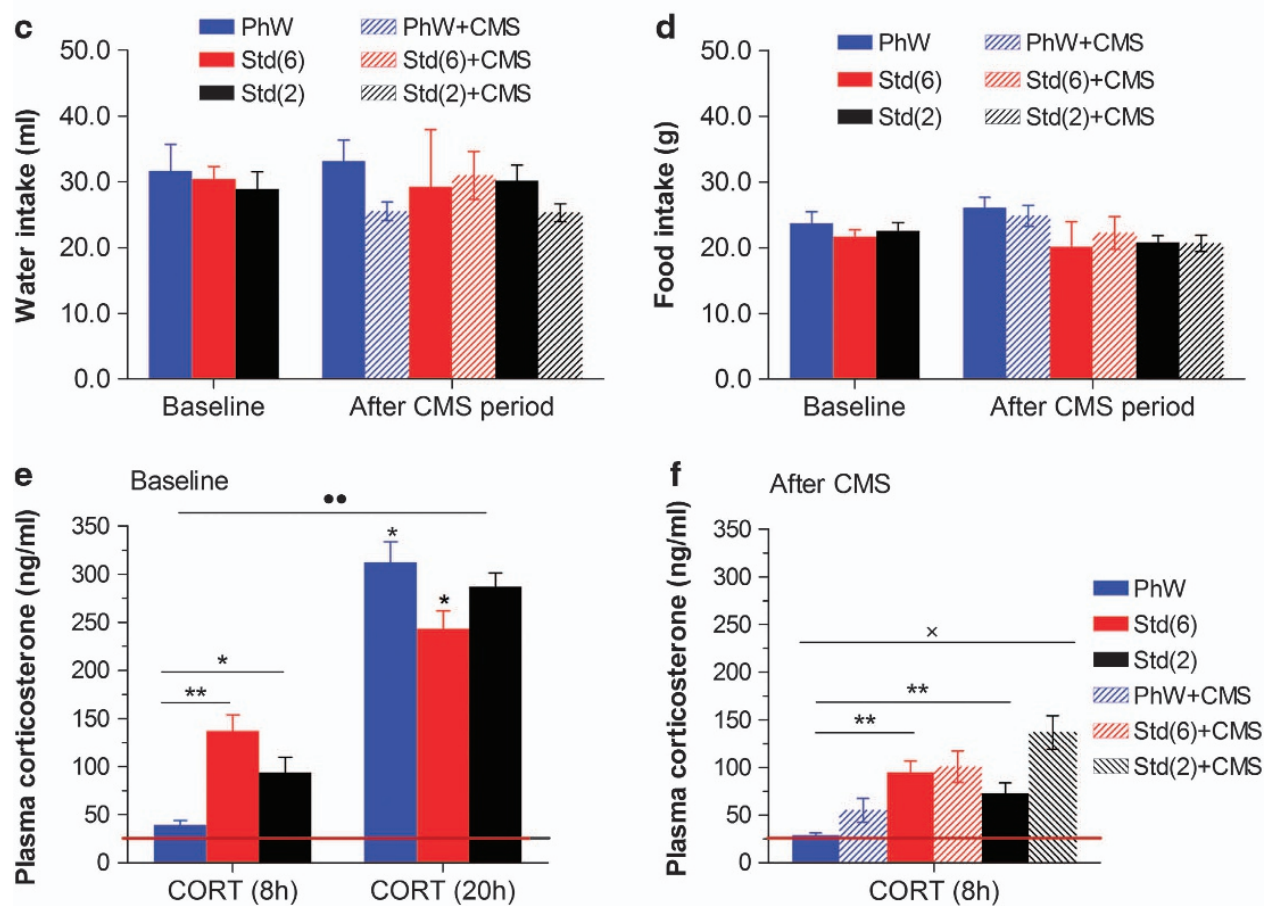

Figure 2. Body weight (a) and body weight variation (b) of animals living in the PhenoWorld (PhW) compared to animals living in standard cages in groups of six or pair-housed (PhW, Std(6) or Std(2) respectively); dashed lines show that animals living in the same conditions lose weight when submitted to unpredictable chronic mild stress (UCMS) protocol (started at week 16-17 of age for PhW+CMS, Std(6)+CMS and Std(2)+CMS groups). The AnimalGates (AG) blue bar indicates the period when animals housed in the PhW adapted to use the AG; the sucrose preference test (SPT-b) gray bar and the uCMS black bar indicate, respectively, the periods when the baseline SPT and the uCMS protocol were performed. (c) Water and (d) food intake per day per animal. (e, f) Plasma corticosterone levels at baseline and six weeks after: CORT (8 h) indicates the time point when lights went on, and CORT $(20 \mathrm{~h})$ indicates the time point when lights went off; the red line on the graphs represents the detection limit for corticosterone measured by radioimmunoassay $\left(25 \mathrm{ng} \mathrm{ml}^{-1}\right)$. Error bars represent s.e.m.; ${ }^{\circ} P \leqslant 0.001$ indicates the effect of time; ${ }^{*} P \leqslant 0.05$ and ${ }^{* *} P \leqslant 0.001$ denote the effect of housing; ${ }^{\times} P \leqslant 0.05$ and ${ }^{x \times} P \leqslant 0.001$ denote the effect of stress. 

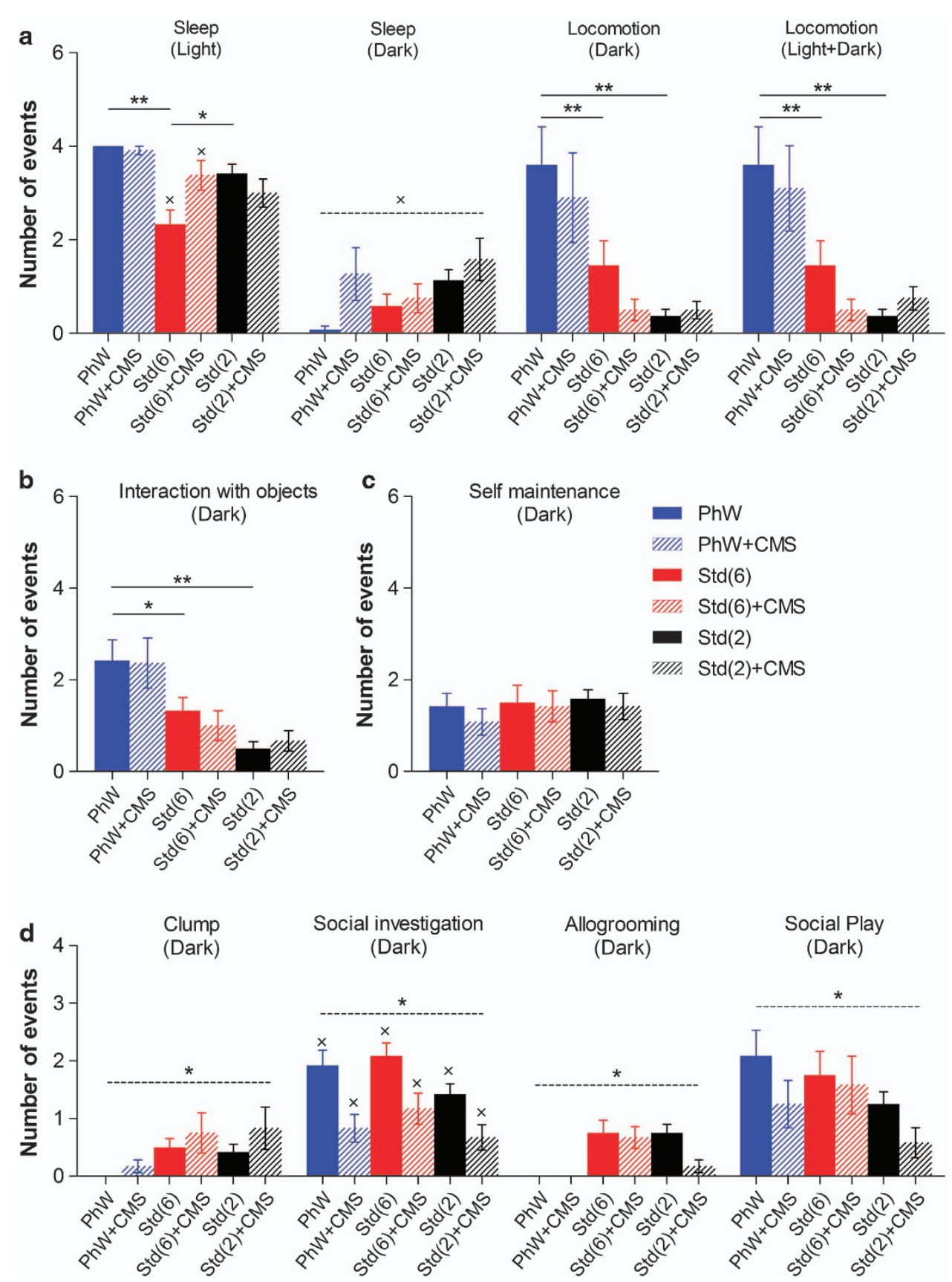

Figure 3. Home-cage behavior: (a) sleeping and locomotion; (b) interaction with objects; (c) self-maintenance (grooming+licking) behavior; and (d) social interaction divided in its different components (clump, social investigation, allogrooming and social play). Error bars represent s.e.m.; ${ }^{*} P \leqslant 0.05$ and ${ }^{* *} P \leqslant 0.001$ denote effects of housing; ${ }^{\times} P \leqslant 0.05$ denote effects of stress. CMS, chronic mild stress; PhW, PhenoWorld.

$20 \mathrm{~h}$, an effect of housing is also shown $\left(\mathrm{F}_{(2,80)}=3.430, P=0.037\right)$ Bonferroni's post hoc indicating a significant difference between PhW animals that had the highest CORT levels at $20 \mathrm{~h}$ and $\mathrm{Std}(6)$ animals $(P=0.035)$. This different pattern resulted in significant differences between the two time points at baseline; one-way ANOVA revealed a significant effect of housing $(F(2,80)=12.986$, $P<0.001$; with PhW animals presenting the highest differences between CORT $(8 \mathrm{~h})$ and CORT $(20 \mathrm{~h}))$; Bonferroni's post hoc test revealed significant differences between $\mathrm{PhW}$ and $\mathrm{Std}(6)$ $(P<0.001)$, between $\mathrm{PhW}$ and $\operatorname{Std}(2)(P=0.028)$, and between $\operatorname{Std}(6)$ and $\operatorname{Std}(2)(P=0.015$; data not shown).

Analysis of plasma corticosterone levels six weeks after baseline revealed and effect of housing $\left(F_{(2,77)}=12.892, P=0.020\right)$ for CORT $(8 \mathrm{~h})$; Bonferroni's post hoc test showed a significant difference between PhW group and both $\operatorname{Std}(6)$ and $\operatorname{Std}(2)$ groups $(P<0.001$ Figure $2 \mathrm{f}$ ) for plasma corticosterone measured at this time point. An effect of stress was also shown $\left(F_{(1,77)}=8.686, P=0.004\right)$, but no interaction effect housing $\times$ stress; further analysis by independent samples $t$-tests performed for each housing condition showed significant differences between control and stressed animals only for pair-housed animals $\left(t_{33}=-3,199 ; P=0,003\right)$, as for animals living in the $\mathrm{PhW}$, the difference did not reach statistical significance $\left(t_{11,7}=-2,067 ; P=0,062\right)$.

Behavioral evaluation

Home-cage behavior. Home-cage behavior scan sampling twoway ANOVA revealed an effect of housing conditions in sleep 
behavior during the light-on period (normal resting phase of the day; $F_{(2,73)}=9.420, P<0.001$; Bonferroni's post hoc test demonstrated that PhW animals slept more than Std(6) animals, $P<0.001$; and Std(2) animals, $P=0.012$ ) during the light-on period. There was no significant effect of stress but a significant interaction housing $\times$ stress in sleeping behavior during the lighton period $\left(\mathrm{F}_{(2,73)}=4.503, P=0.14\right)$. Further analysis comparing control and stressed animals under each housing condition, by independent samples $t$-test, revealed significant differences only between $\operatorname{Std}(6)$ and $\operatorname{Std}(6)+\mathrm{CMS}$ groups $\left(t_{18}=-2.249, P=0.037\right.$; Figure 3a).

During the dark phase (active period of the animals), there was an effect of stress on sleep, as stressed animals slept more than controls $\left(F_{(1,73)}=4.507, P=0.037\right)$, but no effect of housing, nor an interaction housing $\times$ stress effect (Figure $3 a$ ).

The number of locomotion events during the dark period of the light cycle was significantly different between animals in different housing conditions $\left(F_{(2,73)}=19.035, P<0.001\right.$; Bonferroni's post hoc test: $P<0.001$, with animals living in the PhW presenting the higher number, followed by animals living as a group of six in standard cages, and pair-housed animals presenting the lowest number of locomotion events), but there was no effect of stress and no interaction housing $\times$ stress in this behavior.

Analysis of the total locomotion events per day (light+dark period) revealed the same significant differences, reflecting the fact that the dark period is the usual activity period of the animals (housing effect: $F_{(2,73)}=19.600, P=0.001$, with PhW animals presenting higher locomotion compared with $\operatorname{Std}(6)$ or $\operatorname{Std}(2)$ (Bonferroni's post hoc test: $P<0.001$ ); no stress effect: $F_{(1,73)}=0.893, \quad P=0.348$; and no interaction housing $\times$ stress: $\mathrm{F}_{(2,73)}=1.201, P=0.307$; Figure 3a).

Interaction with objects (for example, cardboard tubes) during the animals' active period (dark phase of light cycle) was significantly different as a result of housing conditions $\left(F_{(2,77)}=16.528, P<0.001\right.$; Bonferroni's post hoc test showing a significant difference between $\mathrm{PhW}$ and Std(6) animals, $P=0.002$; and PhW and Std(2) animals, $P<0.001$, with PhW animals presenting higher scores), but no effect of stress nor an interaction housing $\times$ stress could be detected in this behavior (respectively: $F_{(1,77)}=0.078, P=0.781$ and $F_{(2,77)}=0.321, P=0.726$; Figure $3 b$ ).

All animals performed self-maintenance behavior independently of the housing conditions they were living in, or if they had or not been submitted to uCMS protocol (Figure 3c).

Digging was a rare event observed in the dark period of the light cycle and no differences could be observed between groups (data not shown).

For social interaction, we analyzed each social parameter included in this category individually (Figure 3d) during the dark phase of the light cycle. Major contributions were given by events of social investigation and social play, whereas smaller contributions came from events of clumping and allogrooming. Two-way ANOVA showed an effect of housing $\left(\mathrm{F}_{(2,78)}=3.970, P=0.023\right)$, but no significant differences were shown as an effect of stress, as well as no interaction housing $\times$ stress could be detected on clumping
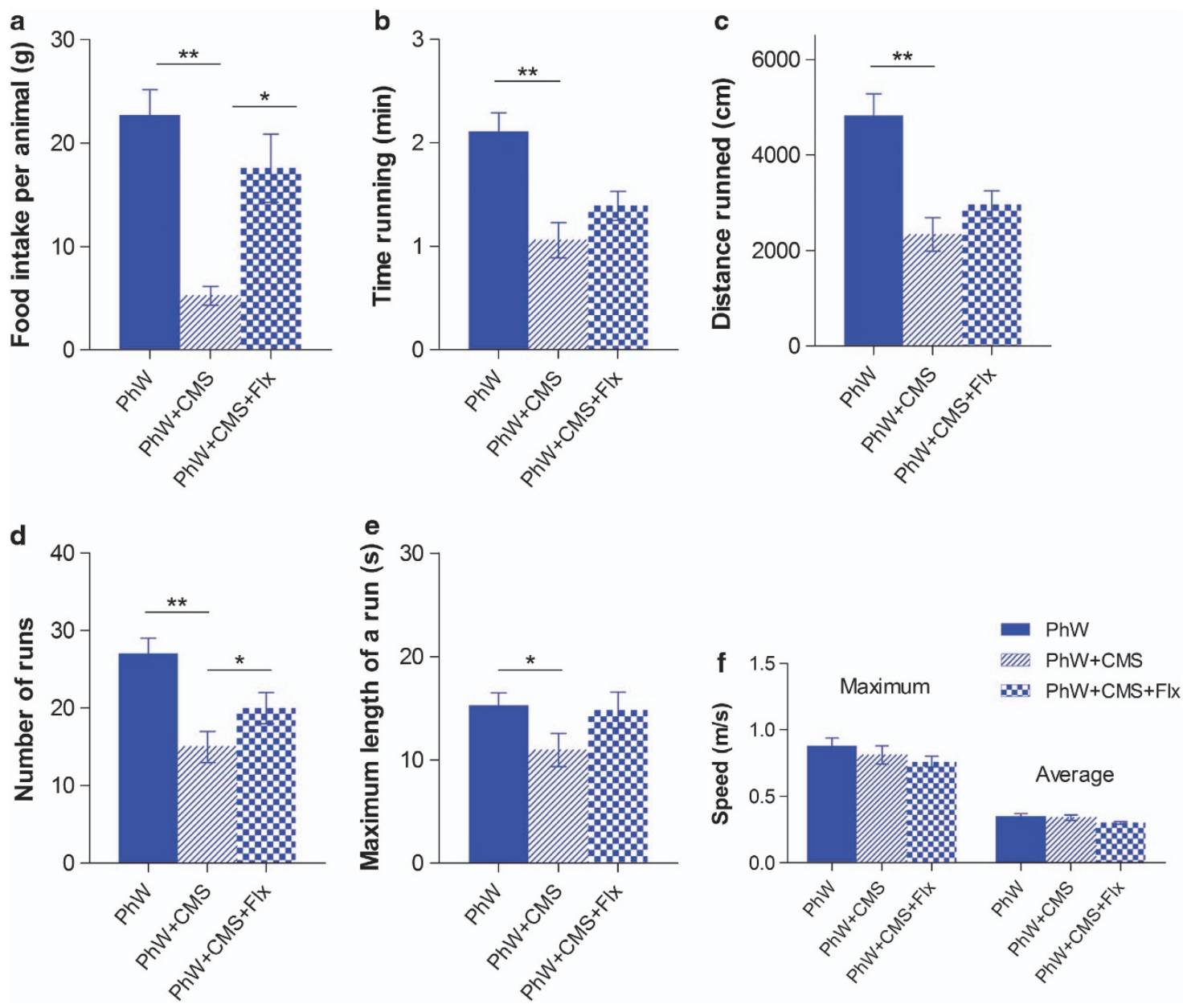

Figure 4. Behaviors automatically registered in the PhenoWorld (PhW) during the dark phase of the light/dark cycle: (a) food intake per animal; (b) time spent in running wheels; (c) distance run in the wheels; (d) number of runs in the wheels; (e) maximum length run in the wheels; and (f) maximum and average speed run in the wheels. Error bars represent s.e.m.; ${ }^{*} P \leqslant 0.05$ and ${ }^{* *} P \leqslant 0.001$. CMS, chronic mild stress; Flx, fluoxetine. 


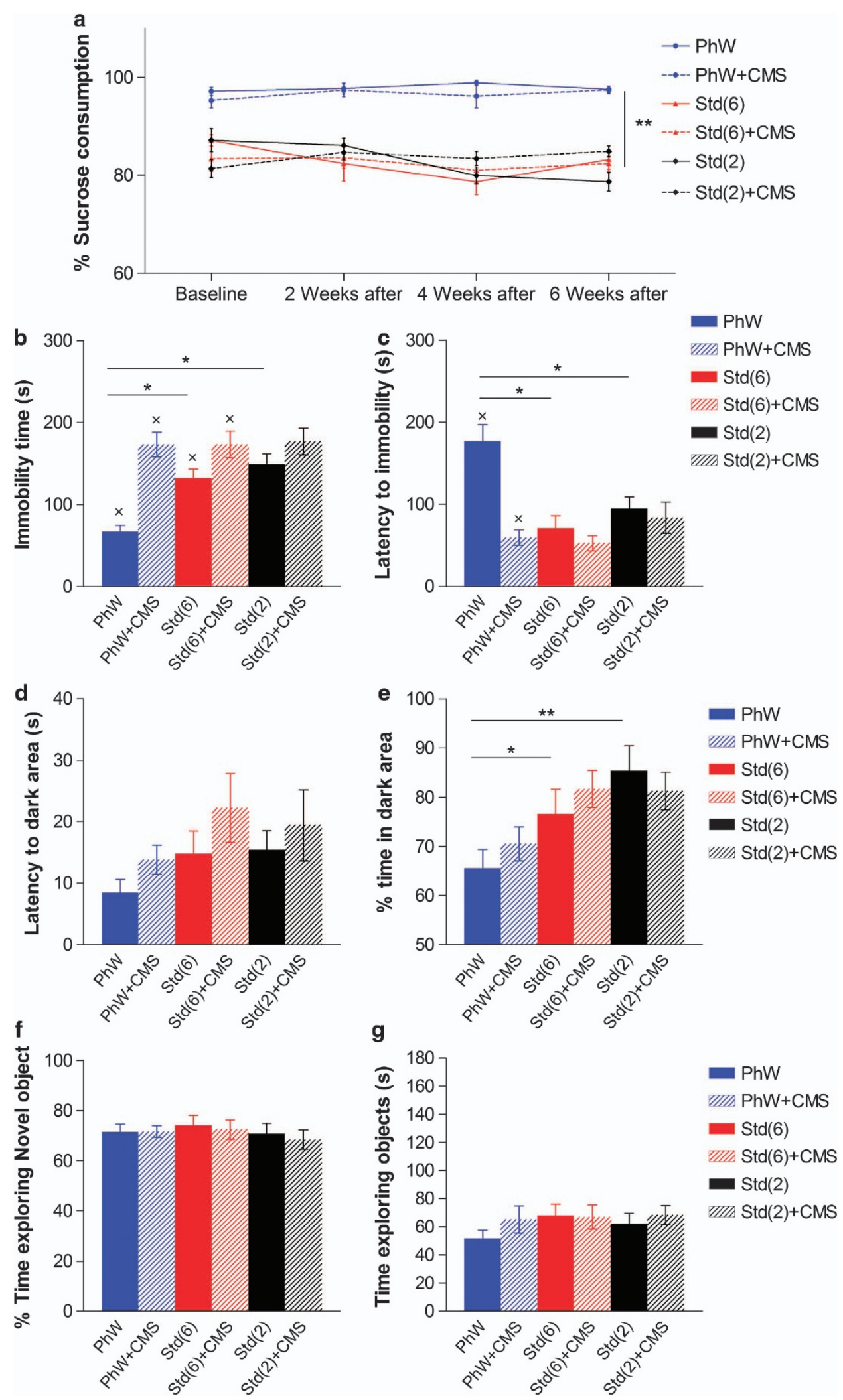

Figure 5. Hedonic behavior was evaluated by a sucrose preference test (a); behavioral despair was evaluated by forced swimming test immobility time (b); latency to immobility (c); anxiety-like behavior in the light/dark box test, measured by latency to the dark area (d); and \% time in the dark area (e). Memory performance evaluated in the novelty object recognition test: (f) presents the percentage of time exploring a novel object; ( $\mathbf{g})$ shows the total time in exploring objects. Error bars represent s.e.m.; ${ }^{*} P \leqslant 0.05$ and ${ }^{* *} P \leqslant 0.001$ denote the effect of housing; ${ }^{x} P \leqslant 0.05$ denote the effect of stress. CMS, chronic mild stress; PhW, PhenoWorld. 
events. Statistical analysis of social investigation showed a significant effect of housing $\left(\mathrm{F}_{(2,78)}=3.235, P=0.045\right)$, with animals living pair-housed presenting the lowest scores; and a significant effect of stress $\left(F_{(1,78)}=22.318, P<0.001\right)$, with stressed animals presenting the lower scores. Independent samples $t$-test revealed significant differences between control and stressed animals in all housing conditions $\left(t_{22}=3.056, P=0.006\right.$ for PhW living animals; $t_{22}=2.586, P=0.017$ for animals living in standard cages of six; and $t_{34}=2.499 P=0.017$ for animals living in standard pair housing). Allogrooming was affected by housing $\left(F_{(2,78)}=9.685, P<0.001\right)$; Bonferroni's post hoc test showed a significant reduction in these events in PhW animals when compared with Std(6) or Std(2) groups $(P \leqslant 0.001)$. There was no significant effect of stress nor a housing $\times$ stress interaction effect on allogrooming. Social play scores analysis showed a significant effect of housing $\left(F_{(2,78)}=3.058, P=0.053\right)$, no significant effect of stress, even though lower scores of social play were shown by animals submitted to uCMS, and no interaction housing $\times$ stress.

For animals living in the PhW, we were able to analyze their willingness to feed and drink and to exercise in running wheels. Figure 4a shows how uCMS significantly decreased food consumption during the active period of the animals $\left(t_{14}=6.582\right.$, $P<0.001)$. The activity in the wheels was also significantly reduced after animals have been submitted to CMS as shown in Figures 4b-e (time running: $t_{21}=4.276, P<0.001$; distance run: $t_{20}=4.276, P<0.001$; number of runs: $t_{20}=4.182, P<0.001$; maximum length achieved in a run: $t_{20}=2.077, P=0.05$ ), whereas their physical ability to run does not seem to be affected as they can achieve similar maximum and average speed when compared with control animals (Figure 4f).

Sucrose and saccharin preference tests. We could observe that animals living in the PhW had a much higher preference for sucrose than animals living in standard conditions either in groups of six or pair-housed (repeated measures ANOVA showed an housing effect: $\left.F_{(2.58)}=37.649, P<0.001\right)$; Bonferroni's post hoc test showing a significant difference between PhW group and both Std(6) and Std( 2$)$ groups $(P<0.001)$, but no significant effect of stress nor interaction housing $\times$ stress could be seen (Figure $5 a$ ). Similar differences in preference could be observed when doing the same test, but using saccharin instead of sucrose (data not shown), which indicates that the preference for sucrose is not driven by energetic needs after fasting, but it is a real increased motivation for drinking sweet solution presented by animals living in the PhW, independently of being submitted to stress or not.

Forced swimming test. Animals living in the PhW displayed increased behavioral despair when compared with animals living in standard conditions as shown in Figure 5b. Two-way ANOVA analysis of immobility time showed an effect of housing $\left(F_{(2,78)}=4.879, P=0.010\right)$, with post hoc analysis revealing significant difference between $\mathrm{PhW}$ group and $\mathrm{Std}(2)$ groups $(P=0.020)$, an effect of stress $\left(F_{(1,78)}=24.850, P<0.001\right)$, and a significant interaction effect of housing $\times$ stress $\left(F_{(2,78)}=4.172\right.$, $P=0.019$ ).

Analysis of latency to immobility (Figure $5 \mathrm{c}$ ) also revealed significant effects of housing $\left(F_{(2,78)}=5.788, P=0.005\right)$ and stress $\left(F_{(1,78)}=14.300, P<0.001\right)$, and a significant interaction effect of housing $\times$ stress $\left(F_{(2,78)}=6.808, P=0.002\right)$; post hoc analysis revealed significant difference between latency to immobility of animals living in the PhW and animals living in standard groups of six animals $(P=0.003)$.

Further comparison of control versus stressed animals from each living condition revealed significant differences between $\mathrm{PhW}$ and $\mathrm{PhW}+\mathrm{CMS}$ groups for both immobility time and latency to immobility $\left(t_{16}=6.318, P<0.001\right.$ and $t_{16}=5.309, P<0.001$, respectively); a significant difference between immobility time between $\operatorname{Std}(6)$ and $\operatorname{Std}(6)+C M S$ animals $\left(t_{22}=2.108, P=0.047\right)$, but no significant difference between these groups for latency to immobility; and no significant differences between Std(2) and Std (2)+CMS animals for any of the two FST parameters.

Light/dark test. Animals living in PhW displayed less anxiety-like behavior as shown in the light/dark box test. No significant effects of housing conditions or stress were found for latency to the dark (Figure $5 \mathrm{~d}$ ), but two-way ANOVA analysis of percentage of time spent in the dark area (Figure 5e) showed an effect of housing $\left(F_{(2,64)}=7.343, P=0.001\right)$, with post hoc analysis revealing significant difference between PhW group and both Std(6) and Std(2) groups ( $P=0.028$ and $P=0.001$, respectively); there was no effect of stress and no significant interaction effect of housing $\times$ stress in this parameter.

Novel object recognition test. The recognition memory of the animals, as tested by the NOR test, was not affected by differences in housing conditions nor by submission of animals to uCMS, as shown by two-way ANOVA of the percentage of time exploring a novel object (Figure 5f). The general exploratory activity of the animals during the NOR test as measured by the total time the animals used in exploring both objects (sample and novel) was also not affected by any of the variables (Figure $5 \mathrm{~g}$ ).

Antidepressant effect. Fluoxetine treatment during the last 2 weeks of UCMS did not reverse the weight loss caused by uCMS (data not shown). Antidepressant treatment also did not have a significant effect on locomotion, interaction with objects or self-maintenance of stressed animals (data not shown).

In contrast, fluoxetine treatment of stressed animals had a significant effect on sleep during the light-on period, increasing the number of sleep events during that period when compared with non-treated stressed rats $\left(F_{(1,48)}=7.726, P=0.008\right.$; not shown in graphs).

Analysis of stressed animals treated with fluoxetine also revealed a reversal of social and feed behaviors and partial recovery of running wheels activity. When analyzing the effects of fluoxetine in stressed animals' home-cage behavior, two-way ANOVA showed a significant housing $\times$ antidepressant interaction effect for social interactions during the active period of the animals $\left(F_{(2,48)}=3.506, P=0.038\right)$. Further analysis by independent samples $t$-test showed a significant increase in these social events in fluoxetine-treated stressed animals living in the PhW, but no significant effect of antidepressant treatment in animals living in standard cages $\left(t_{16}=3.224, P=0.005\right.$ for PhW living animals; $t_{16}=0.823, P=0.423$ for animals living in standard cages of six; and $t_{16}=0.946 P=0.358$ for animals living in standard pair housing).

Interestingly, stressed animals treated with fluoxetine increased their food consumption in the $\mathrm{PhW}\left(t_{12}=3.579, P=0.004\right.$; Figure 4a).

Even though fluoxetine-treated animals tend to present an increased activity in the different parameters related to running wheels behavior, independent samples $t$-test only showed significance in the increase of number of runs performed $\left(t_{19}=2.097, P=0.05\right.$; Figure $\left.4 d\right)$; in the other parameters, there were no significant differences between stressed animals with or without fluoxetine treatment.

Importantly, fluoxetine treatment reverted the effects of stress on behavioral despair. Two-way ANOVA revealed significant effects of housing $\left(F_{(2,48)}=4.632, P=0.014\right)$ and antidepressant $\left(F_{(1.48)}=4.336, P=0.043\right)$, and a significant interaction effect of housing $\times$ antidepressant $\left(F_{(2,48)}=4.651, P=0.014\right)$. Independent samples $t$-test showed a significant antidepressant treatment effect only for stressed animals living in the PhW that recovered from uCMS effect as reflected by the significant decrease in immobility time and increase in latency to immobility $\left(t_{10}=3.647\right.$, $P=0.005$; and $t_{6}=3.385, P=0.017$, respectively; not shown in graphs). 


\section{DISCUSSION}

Housing laboratory rodents in small cages that lack key features of their natural habitats impose constraints on behavior and brain development, ultimately resulting in altered brain functions that can compromise the utility of rodents for research especially in behavioral neuroscience (see Wolfer et al. ${ }^{27}$ for a review). The present study demonstrates that animals living in the PhW, an ethological complex paradigm that better mimics real-life conditions, displayed similar, but more striking, behavioral differences (despair, resting patterns and social behavior) when exposed to uCMS than those displayed by rats living in standard conditions. Moreover, several home-cage behaviors in the PhW, such as willingness to feed and exercise in running wheels, proved to be sensitive indicators of depressive-like behavior.

This study reveals a dual set of advantages in the use of the $\mathrm{PhW}$. The first advantage relates to the ethological value of the paradigm. Indeed, in this setting, there is no unnecessary manipulation of the animals and most measures can be done in the context of their living environment. The latter is relevant, given that it avoids the confounding effect of assessing behavior in a new arena that may produce quite distinct reactions to the novel environment, namely anxiety-like behavior, and affect behavioral performance. Other advantages relate with the ethological value of the PhW as it replicates natural environment conditions much better, even though in the laboratory context, with a more complex and enriched environment than the one created in standard living settings, thereby allowing the animals to perform behaviors they cannot perform in the standard housing conditions (for example, climbing and exercise in the running wheels). This scenario creates a better setting to test for complex behaviors, such as depressive-like behaviors.

The second advantage is the improvement in the quality of the measures produced as a result of living in complex environmental paradigms. $^{26,27}$ Indeed, the present results clearly show that the living environment created by the PhW increase the sensitivity to detect changes in mood behavior with standard tests. As a good illustration of this are the behavior results obtained in the FST and in the observation of social interactions. In the FST, uCMS rats living in the PhW clearly display an increased behavioral despair when compared with animals living in standard conditions, and the impairment in social interactions was higher in the animals that lived in larger groups. Although the first is probably related to the physical (but also emotional) fitness that the enriched environment of the PhW creates, the latter is most likely a result of the much more rich and complex social interactions that occur in living in larger groups with complex social environments. Interestingly, fluoxetine treatment of uCMS rats living in the PhW reversed the effect of stress observed in the FST and in the social interactions. It is important to note at this point that control animals living in the PhW also display less anxiety-like behavior in the light/dark box test, which fits previous observations, ${ }^{28,29}$ and higher sucrose/saccharin preference than those living in standard conditions, but interestingly, in neither of these tests we could observed a significant impact of uCMS. Moreover, we can suggest that the ethological relevance of the PhW is related with both the complexity of the housing and the social enrichment; in fact, social enrichment per se, as tested in groups of similar number of animals living in standard cages, was not enough to cause the same level of sensitivity to detect changes in mood behavior.

Yet, the positive impact in the assessment of depressive-like behavior is not confined to the increased sensitivity of the standard measures, as the PhW also allows for the acquisition of novel measurements that proved to be very sensitive to the effects of uCMS and to the reversal by antidepressants. On this particular point, the data related to willingness to go for food (without any previous food restriction) and for exercise in running wheels, both of which are known to be pleasant to rodents, ${ }^{30,31}$ deserve to be highlighted. Thus, these parameters are likely to represent reliable measurements of hedonic behavior. The fact that for both parameters we found a very significant impact of uCMS clearly supports its validity as sensitive measures of depressive-like behavior; in further support of the validity of these measurements is the reversal obtained with fluoxetine treatment. Finally, it is also of interest to note that the pattern of activity/resting in alignment with the dark/light phases is significantly enhanced in the PhW. This can be taken as a sensitive measure of emotional behavior, given that alterations in these patterns are associated to mood disorders; ${ }^{32,33}$ herein the disruption observed after stress and the reversal with fluoxetine treatment are strongly supportive of its relevance.

In summary, this new paradigm adds significant discriminative and sensitive power to screen depressive-like behavior in rats, in particular hedonic behavior, and will enable future studies to better investigate the mechanisms of complex human diseases such as depression. Given that several other layers of complexity in the assessment of different behavioral domains can be still added in the PhW or that the analysis of longitudinal variations of these measurements is yet to be performed, it is envisaged that this new paradigm will add in the near future valuable insights to phenotypic characterization of mood disorders in animal models.

\section{CONFLICT OF INTEREST}

$\mathrm{HR}$ is an employee of TSE Systems International Group. The remaining authors declare no conflict of interest.

\section{ACKNOWLEDGMENTS}

We specially acknowledge Professor Vera Baumans for the useful discussions and Lars Brauer from TSE Systems International for all his support throughout this project. We thank Drs Ana João Rodrigues, Fernanda Marques, Shilan Aslani and Sónia Borges for their help on specific techniques (blood sampling and RIA) and social behavior analysis; and we thank Knut Eschricht, from TSE Systems International Group, for his technical support in the hardware and software development.

\section{REFERENCES}

1 Kessler RC, Bromet EJ. The epidemiology of depression across cultures. Annu Rev Public Health 2013; 34: 119-138.

2 WHO . Depression, A Global Public Health Concern. 2012, pp 6-8.

3 Pollak DD, Rey CE, Monje FJ. Rodent models in depression research: classical strategies and new directions. Ann Med 2010; 42: 252-264.

4 Sousa N, Almeida OFX, Wotjak CT. A hitchhiker's guide to behavioral analysis in laboratory rodents. Genes Brain Behav 2006; 5(Suppl 2): 5-24.

5 Bessa JM, Mesquita AR, Oliveira M, Pêgo JM, Cerqueira JJ, Palha JA et al. A transdimensional approach to the behavioral aspects of depression. Front. Behav Neurosci 2009; 3: 1 .

6 Andreatini R, Bacellar LF. Animal models: trait or state measure? The test-retest reliability of the elevated plus-maze and behavioral despair. Prog Neuropsychopharmacol Biol Psychiatry 2000; 24: 549-560.

7 Balcombe JP, Barnard ND, Sandusky C. Laboratory routines cause animal stress. Contemp Top Lab Anim Sci 2004; 43: 42-51.

8 Castelhano-Carlos MJ, Baumans V. The impact of light, noise, cage cleaning and in-house transport on welfare and stress of laboratory rats. Lab Anim 2009; 43 311-327.

9 Rygula R, Abumaria N, Flügge G, Fuchs E, Rüther E, Havemann-Reinecke U. Anhedonia and motivational deficits in rats: impact of chronic social stress. Brain Res 2005; 162: 127-134

10 Van Driel KS, Talling JC. Familiarity increases consistency in animal tests. Behav Behav Brain Res 2005; 159: 243-245.

11 Willner P. Validity, reliability and utility of the chronic mild stress model of depression: a 10-year review and evaluation. Psychopharmacology (Berl) 1997; 134: 319-329.

12 Willner P. Chronic mild stress (CMS) revisited: consistency and behaviouralneurobiological concordance in the effects of CMS. Neuropsychobiology 2005; 52: 90-110.

13 Nicklas W, Baneux P, Boot R, Decelle T, Deeny AA, Fumanelli M et al. Recommendations for the health monitoring of rodent and rabbit colonies in breeding and experimental units. Lab Anim 2002; 36: 20-42. 
14 Bessa JM, Ferreira D, Melo I, Marques F, Cerqueira JJ, Palha JA et al. The moodimproving actions of antidepressants do not depend on neurogenesis but are associated with neuronal remodeling. Mol Psychiatry 2009; 14: 764-773, 739.

15 Abou-Ismail UA, Burman OHP, Nicol CJ, Mendl M. The effects of enhancing cage complexity on the behaviour and welfare of laboratory rats. Behav Processes 2010; 85: $172-180$.

16 Vanderschuren LJ, Niesink RJ, Van Ree JM. The neurobiology of social play behavior in rats. Neurosci Biobehav Rev 1997; 21: 309-326.

17 Bekris S, Antoniou K, Daskas S, Papadopoulou-Daifoti Z. Behavioural and neurochemical effects induced by chronic mild stress applied to two different rat strains. Behav Brain Res 2005; 161: 45-59.

18 Smith JC, Sclafani A. Saccharin as a sugar surrogate revisited. Appetite 2002; 38: $155-160$.

19 Grønli J, Murison R, Fiske E, Bjorvatn B, Sørensen E, Portas CM et al. Effects of chronic mild stress on sexual behavior, locomotor activity and consumption of sucrose and saccharine solutions. Physiol Behav 2005; 84: 571-577.

20 Dalla C, Pitychoutis PM, Kokras N, Papadopoulou-Daifoti Z. Sex differences in animal models of depression and antidepressant response. Basic Clin Pharmacol Toxicol 2010; 106: 226-233.

21 Castagné V, Moser P, Roux S, Porsolt RD. Rodent models of depression: forced swim and tail suspension behavioral despair tests in rats and mice. Curr Protoc Neurosci 2001; 14: 8.10A.1-8.10A.10.

22 Cryan JF, Markou A, Lucki I. Assessing antidepressant activity in rodents: recent developments and future needs. Trends Pharmacol. Sci. 2002; 23: 238-245.

23 Hascoët M, Bourin M, Dhonnchadha BA. The mouse light-dark paradigm: a review. Prog Neuropsychopharmacol Biol Psychiatry 2001; 25: 141-166.

24 Huang $\mathrm{T}-\mathrm{Y}$, Lin $\mathrm{C}-\mathrm{H}$. A comparison between chronic exercise training and desipramine as treatments for the depression-like behavior of early-life maternal deprivation rats. Neurosci Lett 2010; 480: 201-205.
25 Bevins RA, Besheer J. Object recognition in rats and mice: a one-trial nonmatching-to-sample learning task to study 'recognition memory'. Nat Protoc 2006; 1: 1306-1311.

26 Balcombe JP. Laboratory environments and rodents' behavioural needs: a review. Lab Anim 2006; 40: 217-235.

27 Wolfer DP, Litvin O, Morf S, Nitsch RM, Lipp H-P, Würbel H. Laboratory animal welfare: cage enrichment and mouse behaviour. Nature 2004; 432: 821-822.

28 Sztainberg Y, Chen A. An environmental enrichment model for mice. Nat Protoc 2010; 5: 1535-1539.

29 Sampedro-Piquero P, Zancada-Menendez C, Begega A, Rubio S, Arias JL. Effects of environmental enrichment on anxiety responses, spatial memory and cytochrome $c$ oxidase activity in adult rats. Brain Res Bull 2013; 98: 1-9.

30 Saper CB, Chou TC, Elmquist JK. The need to feed: homeostatic and hedonic control of eating. Neuron 2002; 36: 199-211.

31 Greenwood BN, Foley TE, Le T V, Strong P V, Loughridge AB, Day HEW et al. Longterm voluntary wheel running is rewarding and produces plasticity in the mesolimbic reward pathway. Behav Brain Res 2011; 217: 354-362.

32 McClung CA. Circadian rhythms and mood regulation: insights from preclinical models. Eur Neuropsychopharmacol 2011; 21(Suppl 4): S683-S693.

33 Savelyev SA, Rantamäki T, Rytkönen K-M, Castren E, Porkka-Heiskanen T. Sleep homeostasis and depression: studies with the rat clomipramine model of depression. Neuroscience 2012; 212: 149-158.

(c) (i) $\Theta$ This work is licensed under a Creative Commons AttributionBY NC ND NonCommercial-NoDerivs 3.0 Unported License. The images or other third party material in this article are included in the article's Creative Commons license, unless indicated otherwise in the credit line; if the material is not included under the Creative Commons license, users will need to obtain permission from the license holder to reproduce the material. To view a copy of this license, visit http://creativecommons.org/licenses/by-nc-nd/3.0/

Supplementary Information accompanies the paper on the Translational Psychiatry website (http://www.nature.com/tp) 